\title{
Infinite Horizon LQ Zero-Sum Stochastic Differential Games with Markovian Jumps
}

\author{
Huai-Nian Zhu ${ }^{1}$, Cheng-Ke Zhang ${ }^{2}$, Ning Bin ${ }^{1}$ \\ ${ }^{1}$ School of Management, Guangdong University of Technology, Guangzhou, China \\ ${ }^{2}$ School of Economics \& Commence, Guangdong University of Technology, Guangzhou, China \\ Email: huainian258@163.com
}

Received June 22, 2012; revised July 22, 2012; accepted July 30, 2012

\begin{abstract}
This paper studies a class of continuous-time two person zero-sum stochastic differential games characterized by linear Itô's differential equation with state-dependent noise and Markovian parameter jumps. Under the assumption of stochastic stabilizability, necessary and sufficient condition for the existence of the optimal control strategies is presented by means of a system of coupled algebraic Riccati equations via using the stochastic optimal control theory. Furthermore, the stochastic $H_{\infty}$ control problem for stochastic systems with Markovian jumps is discussed as an immediate application, and meanwhile, an illustrative example is presented.
\end{abstract}

Keywords: Stochastic Systems; Differential Games; Markovian Jumps; Stochastic $H_{\infty}$ Control

\section{Introduction}

The stochastic control problems governed by Itô's differential equation have become a popular research topic in past decades. Recently, stochastic $H_{\infty}$ control problem with state and control—-dependent noise was considered $[1,2]$. It has attracted much attention and has been widely applied to various fields. Particularly, the stochastic $\mathrm{H}_{2} / \mathrm{H}_{\infty}$ control with state-dependent noise has been addressed $[3,4]$. Recently, linear quadratic differential games and their applications have been widely investigated in many literatures, and examples of differential games in economics and management science can be found e.g. in [5-9]. These results are mainly based on the deterministic systems. However, to the best of our knowledge, few results have been obtained for stochastic differential games with Markovian jumps.

In this paper, the stochastic zero-sum games for linear quadratic systems governed by Itô's differential equations with state-dependent noise and Markovian jumps are addressed, Such class of systems has important applications in engineering practice since they can be used to represent random failure processes in manufacturing systems, electric power systems and so on, see [10-18]. In particular, stability and robust stabilization for such perturbed systems were investigated extensively in [13, 15,17]. A bounded real lemma for Markovian jump stochastic systems was derived in [13]. [11] studied the optimal filtering problem for such systems, while $[12,14,16]$ addressed the issue of linear quadratic regulator. The goal of this paper is to develop the differential game theory for stochastic Itô systems with Markovian jumps, a necessary and sufficient condition is developed for the existence of optimal control strategies in terms of a coupled algebraic Riccati equations (AREs), which can be viewed as an extension of the existing results of [19]. In the end, stochastic $H_{\infty}$ control problem with Markovian jumps is given as our theoretical applications and an illustrative example is presented.

For convenience, we will make use of the following notations in this paper:

$A^{T}$ : transpose of a matrix or vector $A ; A^{-1}$ : inverse of a matrix or vector $A ; A>0(A \geq 0)$ : positive definite (positive semidefinite) symmetric matrix $A ; \chi_{A}$ : indicator function of a set $A ; M_{n, m}^{l}$ : space of all $A=(A(1), A(2), \cdots, A(l))$ with $A(i)$ being $n \times m$ matrix, $i=1,2, \cdots, l ; M_{n}^{l}:=M_{n, n}^{l} ; \mathbb{S}_{n}:$ space of all $n \times n$ symmetric matrices; $\mathbb{S}_{n}^{l}$ : space of all

$A=(A(1), A(2), \cdots, A(l))$ with $A(i)$ being $n \times n$ symmetric matrix, $i=1,2, \cdots, l$;

$A=(A(1), A(2), \cdots, A(l))>0 \quad(\geq 0)$ means $A(i)>0$ $(\geq 0)$ for $i=1,2, \cdots, l ; \mathbb{R}^{n}$ : space of all $n$-dimensional real vectors with usual 2 -norm $|\bullet|$.

\section{Definitions and Preliminaries}

Throughout this paper, let $\left(\Omega, F,\left\{F_{t}\right\}_{t \geq 0}, P\right)$ be a given filtered probability space where there exists a standard one dimensional Wiener process $\{w(t)\}_{t>0}$, and a right continuous homogeneous Markov chain $\left\{r_{t}\right\}_{t \geq 0}$ with state 
space $\varphi=\{1,2, \cdots, l\}$. We assume that $\left\{r_{t}\right\}$ is independent of $\{w(t)\}$ and has the following transition probability:

$$
\operatorname{Pr}\left\{r_{t+\Delta}=j \mid r_{t}=i\right\}= \begin{cases}\pi_{i j} \Delta+o(\Delta), & i \neq j \\ 1+\pi_{i i} \Delta+o(\Delta), & i=j\end{cases}
$$

where $\pi_{i j} \geq 0$ for $i \neq j$ and $\pi_{i i}=-\sum_{i \neq j} \pi_{i j} . F_{t}$ stands for the smallest $\sigma$-algebra generated by process $w(s), r_{s}$, $0 \leq s \leq t$, i.e. $F_{t}=\sigma\left\{w(s), r_{s} \mid 0 \leq s \leq t\right\}$.

By $L_{F}^{2}\left([0, \infty) ; \mathbb{R}^{m}\right)$ denote the space of all measureable functions $u(t, x):=[0, \infty) \times \mathbb{R}^{n} \mapsto \mathbb{R}^{m}$, which is $F_{t}$ -measurable for every $t \geq 0$, and

$E\left[\int_{0}^{\infty}|u(t)|^{2} \mathrm{~d} t \mid r_{0}=i\right]<\infty, \quad i \in \varphi$. Obviously,

$L_{F}^{2}\left([0, \infty) ; \mathbb{R}^{m}\right)$ is a Hilbert space with the inner product

$$
\langle u, v\rangle=\sum_{i=1}^{l} E\left[\int_{0}^{\infty} u^{\mathrm{T}}(t) v(t) \mathrm{d} t \mid r_{0}=i\right]
$$

Consider the following linear stochastic controlled system with Markovian jumps

$$
\mathrm{d} x(t)=\left[A\left(r_{t}\right) x(t)+B\left(r_{t}\right) u(t)\right] \mathrm{d} t+C\left(r_{t}\right) x(t) \mathrm{d} t
$$

where $x(t) \in \mathbb{R}^{n}$ and $u(t) \in \mathbb{R}^{m}$ are the state and control input, respectively. The coefficients $A, C \in M_{n}^{l}$ and $B \in M_{n, m}^{l}$ with $A(i), B(i), C(i), i \in \varphi$, being constant matrices.

It is well known that for any $u(t) \in L_{F}^{2}\left([0, \infty) ; \mathbb{R}^{m}\right)$ and $\left(x_{0}, i\right) \in \mathbb{R}^{n} \times \varphi$, there exists a unique solution $x(t) \in L_{F}^{2}\left([0, \infty) ; \mathbb{R}^{n}\right)$ of (1) with initial condition $x(0)=x_{0}, r_{0}=i$. Next, we first introduce the definition of stochastic stabilizability which is an essential assumption in this paper.

Definition 1. System (2) or $(A, B, C)$ is called stochastic stabilizable (in mean-square sense), if there exists a feedback control $u(t)=\sum_{i=1}^{l} K(i) x(t) \chi_{r_{t}=i}$ with $K(1), K(2), \cdots, K(l)$ being constant matrices, such that for any initial state $x(0)=x_{0}, r_{0}=i$, the closed-loop system

$$
\mathrm{d} x(t)=\left[A\left(r_{t}\right)+B\left(r_{t}\right) K\left(r_{t}\right)\right] x(t) \mathrm{d} t+C\left(r_{t}\right) x(t) \mathrm{d} t
$$

is asymptotically mean-square stable, i.e.

$$
\lim _{t \rightarrow \infty} E\left[x^{T}(t) x(t) \mid r_{0}=i\right]=0
$$

Now we give two lemmas which are important in our subsequent analysis. For system (2), by applying Itô's formula to $x^{T} P(i) x$, we immediately obtain the following result.
Lemma 1. Suppose $P=(P(1), P(2), \cdots, P(l)) \in \mathbb{S}_{n}^{l}$ is given, then for system (2) with initial condition $\left(x_{0}, i\right) \in \mathbb{R}^{n} \times \varphi$, we have (see Equation (3) below)

Lemma 2 [4]. For system (2), $(A, B, C)$ is stochastic stabilizable if and only if (iff) the following Lyapunov-type equation:

$$
\begin{aligned}
L(P):= & P(i) A(i)+A^{\mathrm{T}}(i) P(i)+A_{1}^{\mathrm{T}}(i) P(i) A_{1}(i) \\
& +\sum_{j=1}^{l} \pi_{i j} P(j)+C^{\mathrm{T}}(i) C(i)=0, i \in \varphi
\end{aligned}
$$

has a unique positive semidefinite solution $P=(P(1), P(2), \cdots P(l)) \in \mathbb{S}_{n}^{l}$.

\section{Problem Formulation}

Fix $\left(0, x_{0}\right) \in[0, \infty) \times \mathbb{R}^{n}$. Let $U_{\tau}[0, \infty)$ be the set of the $\mathbb{R}^{k}$-valued, square integrable processes adapted with the $\sigma$-field generated by $w(t), r_{t}, \tau=1,2$, respectively. Associated with each $\left(u_{1}, u_{2}\right) \in U[0, \infty) \equiv U_{1}[0, \infty) \times U_{2}[0, \infty)$ is a quadratic cost functional $J\left(u_{1}, u_{2} ; x_{0}, i\right)$ :

$$
\begin{aligned}
J\left(u_{1}, u_{2} ; x_{0}, i\right) \\
=E\left\{\int _ { 0 } ^ { \infty } \left[x^{\mathrm{T}}(t) Q\left(r_{t}\right) x(t)+2 x^{\mathrm{T}}(t) S_{1}\left(r_{t}\right) u_{1}(t)\right.\right. \\
+u_{1}^{\mathrm{T}}(t) R_{1}\left(r_{t}\right) u_{1}(t)+2 x^{\mathrm{T}}(t) S_{2}\left(r_{t}\right) u_{2}(t) \\
\left.\left.+u_{2}^{\mathrm{T}}(t) R_{2}\left(r_{t}\right) u_{2}(t)\right] \mathrm{d} t \mid r_{0}=i\right\}
\end{aligned}
$$

where $R_{1}\left(r_{t}\right)>0, R_{2}\left(r_{t}\right)<0, Q\left(r_{t}\right) \geq 0, S_{1}\left(r_{t}\right) \geq 0$, $S_{2}\left(r_{t}\right) \geq 0, E\{\bullet\}$ represents the expectation of the enclosed random variable, $x(t)$ is the solution to the following linear stochastic differential equation with statedependent noise and Markovian parameter jumps

$$
\begin{aligned}
\mathrm{d} x(t)= & {\left[A\left(r_{t}\right) x(t)+B_{1}\left(r_{t}\right) u_{1}(t)+B_{2}\left(r_{t}\right) u_{2}(t)\right] \mathrm{d} t } \\
& +C\left(r_{t}\right) x(t) \mathrm{d} w(t), x(0)=x_{0}
\end{aligned}
$$

In (5) and (6), $A\left(r_{t}\right)=A_{i}$, etc. whenever $r_{t}=i$. Now we consider the following zero-sum differential game problem.

Problem 1. Given a system described by (6), find $\left(u_{1}^{*}, u_{2}^{*}\right) \in U[0, \infty)$, such that

$$
\begin{aligned}
\underset{u_{2}}{\operatorname{Max}} \operatorname{Min}_{u_{1}} J\left(u_{1}, u_{2} ; x_{0}, i\right) & =\underset{u_{1}}{\operatorname{Min}} \operatorname{Max} J\left(u_{1}, u_{2} ; x_{0}, i\right) \\
& =J\left(u_{1}^{*}, u_{2}^{*} ; x_{0}, i\right),
\end{aligned}
$$

$$
\begin{gathered}
E\left\{\int _ { 0 } ^ { \infty } \left[x^{\mathrm{T}}(t)\left(P\left(r_{t}\right) A\left(r_{t}\right)+A^{\mathrm{T}}\left(r_{t}\right) P\left(r_{t}\right)+C^{\mathrm{T}}\left(r_{t}\right) P\left(r_{t}\right) C\left(r_{t}\right)+\sum_{j=1}^{l} \pi_{r_{i} j} P(j)\right) x(t)\right.\right. \\
\left.\left.+2 u^{\mathrm{T}}(t) B^{\mathrm{T}}\left(r_{t}\right) P\left(r_{t}\right) x(t)\right] \mathrm{d} t \mid r_{0}=i\right\}=-x_{0}^{\mathrm{T}} P(i) x_{0}
\end{gathered}
$$


or equivalently,

$$
\begin{gathered}
J\left(u_{1}^{*}, u_{2} ; x_{0}, i\right) \leq J\left(u_{1}^{*}, u_{2}^{*} ; x_{0}, i\right) \leq J\left(u_{1}, u_{2}^{*} ; x_{0}, i\right), \\
\forall\left(u_{1}, u_{2}\right) \in U[0, \infty) .
\end{gathered}
$$

That is, there are two players for the differential game. Player 1 chooses control $u_{1}^{*}$ to minimize the objective $J$, while Player 2 chooses control $u_{2}^{*}$ to maximize $J$. Now we introduce a new type of coupled algebraic Riccati equations associated with the problem 1.

Definition 2. The following system of algebraic equations

$$
\begin{aligned}
& P(i) A(i)+A^{\mathrm{T}}(i) P(i)+C^{\mathrm{T}}(i) P(i) C(i)+Q(i) \\
& +\sum_{j=1}^{l} \pi_{i j} P(j)-\left(P(i) B(i)+S^{\mathrm{T}}(i)\right) \\
& \times R^{-1}(i)\left(B^{\mathrm{T}}(i) P(i)+S(i)\right)=0, i \in \varphi
\end{aligned}
$$

with

$$
B(i)=\left(B_{1}(i), B_{2}(i)\right), S(i)=\left(\begin{array}{l}
S_{1}^{\mathrm{T}}(i) \\
S_{2}^{\mathrm{T}}(i)
\end{array}\right), R(i)=\left(\begin{array}{cc}
R_{1}(i) & 0 \\
0 & -R_{2}(i)
\end{array}\right) .
$$

is called a system of coupled algebraic Riccati equations (AREs).

In the next section, we will give our main results of

$$
\begin{aligned}
& J\left(u_{1}, u_{2} ; x_{0}, i\right) \\
&=x_{0}^{\mathrm{T}} P(i) x_{0}+E\left\{\int _ { 0 } ^ { \infty } \left[x ^ { \mathrm { T } } ( t ) \left(P\left(r_{t}\right) A\left(r_{t}\right)+A^{\mathrm{T}}\left(r_{t}\right) P\left(r_{t}\right)+Q\left(r_{t}\right)\right.\right.\right. \\
&+\left.C^{\mathrm{T}}\left(r_{t}\right) P\left(r_{t}\right) C\left(r_{t}\right)+\sum_{j=1}^{l} \pi_{r_{t} j} P(j)\right) x(t)+u_{1}^{\mathrm{T}}(t) R_{1}\left(r_{t}\right) u_{1}(t) \\
&+ 2 u_{1}^{\mathrm{T}}(t)\left(B_{1}^{\prime}\left(r_{t}\right) P\left(r_{t}\right)+S_{1}^{\prime}\left(r_{t}\right)\right) x(t)+u_{2}^{\mathrm{T}}(t) R_{2}\left(r_{t}\right) u_{2}(t) \\
&\left.\left.+2 u_{2}^{\mathrm{T}}(t)\left(B_{2}^{\prime}\left(r_{t}\right) P\left(r_{t}\right)+S_{2}^{\prime}\left(r_{t}\right)\right) x(t)\right] \mathrm{d} t \mid r_{0}=i\right\} .
\end{aligned}
$$

Proof. Sufficiency: Let $P=(P(1), P(2), \cdots, P(l)) \in \mathbb{S}_{n}^{l}$ be a solution of the AREs (7). According to lemma 1, we have

By a series of simple computation together with (7), the cost function $J\left(u_{1}, u_{2} ; x_{0}, i\right)$ can be expressed as following

$$
\begin{aligned}
J\left(u_{1}, u_{2} ; x_{0}, i\right) & \\
=x_{0}^{\mathrm{T}} P(i) x_{0}+ & E\left\{\int _ { 0 } ^ { \infty } \left[\left(u_{1}(t)-u_{1}^{*}(t)\right)^{\mathrm{T}} R_{1}\left(r_{t}\right)\left(u_{1}(t)-u_{1}^{*}(t)\right)\right.\right. \\
& \left.\left.+\left(u_{2}(t)-u_{2}^{*}(t)\right)^{\mathrm{T}} R_{2}\left(r_{t}\right)\left(u_{2}(t)-u_{2}^{*}(t)\right)\right] \mathrm{d} t \mid r_{0}=i\right\} .
\end{aligned}
$$

Thus, $J\left(u_{1}, u_{2} ; x_{0}, i\right)$ is minimized by the control strategies $u_{1}^{*}(t)$ and $u_{2}^{*}(t)$ with the optimal value being $x_{0}^{T} P(i) x_{0}$.

Necessity: Let

$u_{1}^{*}(t)=K_{1}\left(r_{t}\right) x(t)=-R_{1}^{-1}\left(r_{t}\right)\left(B_{1}^{\mathrm{T}}\left(r_{t}\right) P\left(r_{t}\right)+S_{1}\left(r_{t}\right)\right) x(t)$,

$u_{2}^{*}(t)=K_{2}\left(r_{t}\right) x(t)=-R_{2}^{-1}\left(r_{t}\right)\left(B_{2}^{\mathrm{T}}\left(r_{t}\right) P\left(r_{t}\right)+S_{2}\left(r_{t}\right)\right) x(t)$

be the optimal control strategies to problem 1. Implement $u_{1}^{*}(t)=K_{1}\left(r_{t}\right) x(t)$ and $u_{2}^{*}(t)=K_{2}\left(r_{t}\right) x(t)$ in (6), then

$$
\mathrm{d} x(t)=\tilde{A}\left(r_{t}\right) x(t) \mathrm{d} t+C\left(r_{t}\right) x(t) \mathrm{d} w(t), x(0)=x_{0}
$$

where

$$
\begin{aligned}
\tilde{A}\left(r_{t}\right)= & A\left(r_{t}\right)-B_{1}\left(r_{t}\right) R_{1}^{-1}\left(r_{t}\right)\left(B_{1}^{\mathrm{T}}\left(r_{t}\right) P\left(r_{t}\right)+S_{1}\left(r_{t}\right)\right) \\
& -B_{2}\left(r_{t}\right) R_{2}^{-1}\left(r_{t}\right)\left(B_{2}^{\mathrm{T}}\left(r_{t}\right) P\left(r_{t}\right)+S_{2}\left(r_{t}\right)\right)
\end{aligned}
$$

According to lemma 2 and the stochastic optimal control theory, we can easily obtain the conclusion that the AREs (7) admit a solution $P=(P(1), P(2), \cdots, P(l)) \in \mathbb{S}_{n}^{l}$. 
So this completes the proof of Theorem 1.

Remark 1. It is interesting to see the specialization of our results in the deterministic case (i.e. $C(i) \equiv 0$ for $i \in \varphi)$. The corresponding AREs are

$$
\begin{gathered}
P(i) A(i)+A^{\mathrm{T}}(i) P(i)+Q(i)+\sum_{j=1}^{l} \pi_{i j} P(j) \\
-\left(P(i) B(i)+S^{\mathrm{T}}(i)\right)^{\mathrm{T}} R^{-1}(i)\left(B^{\mathrm{T}}(i) P(i)+S(i)\right)=0,(8) \\
i \in \varphi .
\end{gathered}
$$

which can be viewed as an extended results of [12].

Remark 2. From Theorem 1 we can see that the derivation of the optimal control strategies for this type of differential games is transformed into deriving the solutions to coupled algebraic Riccati Equations (7), this conclusion be coincident with the results presented in [4], etc.

Remark 3. For the coupled algebraic Riccati Equations (7) may be solved by a standard numerical integration such as LMI method [20], or iterative algorithm similar with the algorithm presented in [21].

\section{Application to Stochastic $\boldsymbol{H}_{\infty}$ Control}

Now, we apply the above developed theory to solve some problems related to stochastic $H_{\infty}$ control. Firstly, we statement the stochastic $H_{\infty}$ control problem with Markovian jumps, then, we demonstrate the usefulness of the above developed theory in the study of stochastic $H_{\infty}$ control.

Consider the following controlled system:

$$
\begin{aligned}
\mathrm{d} x(t)= & {\left[A\left(r_{t}\right) x(t)+B_{1}\left(r_{t}\right) v(t)+B_{2}\left(r_{t}\right) u(t)\right] \mathrm{d} t } \\
& +C\left(r_{t}\right) x(t) \mathrm{d} w(t)
\end{aligned}
$$

with the cost functional

$$
J(u)=E\left\{\int_{0}^{\infty}\left[x^{\mathrm{T}}(t) Q\left(r_{t}\right) x(t)+|u(t)|^{2}-\gamma^{2}|v(t)|^{2}\right] \mathrm{d} t\right\}(10)
$$

where $\left\{r_{t}\right\}_{t \geq 0}$ is a right continuous Markov process on a given probability space $\left(\Omega, F,\left\{F_{t}\right\}_{t \geq 0}, P\right)$ and the state space $\varphi=\{1,2, \cdots, l\}$ and the transition probability described by (1); here $\{w(t)\}_{t \geq 0}$ is a standard one dimensional Wiener processes. In (9) and (10), $x(t) \in \mathbb{R}^{n}$ is the state vector, $u(t) \in \mathbb{R}^{m_{2}}$ is the input control and $v(t) \in \mathbb{R}^{m_{1}}$ is the vector of the exogenous disturbances.

The following definition is parallel with the definition 2 presented in [22].

Definition 3. Given disturbance attenuation level $\gamma>0$, the state feedback strategy

$u^{*}(t)=u^{*}\left(x, r_{t}\right) \in L_{F}^{2}\left([0, \infty), \mathbb{R}^{m_{2}}\right)$ is said to be an $H_{\infty}$ control for system (9), if for $x(0)=x_{0}, r_{0}=i \in \varphi$, $\forall v(t) \in L_{F}^{2}\left([0, \infty) \mathbb{R}^{m_{1}}\right)$, we have

1) $u^{*}(t)$ stabilizes system (9) internally, i.e. when $v(t)=0, u=u^{*}$, the state trajectory of (9) with any initial value $\left(x_{0}, i\right) \in \mathbb{R}^{n} \times \varphi$ satisfies

$$
\lim _{t \rightarrow \infty} E\left[x^{\mathrm{T}}(t) x(t) \mid r_{0}=i\right]=0
$$

2) $\left|L_{u^{*}}\right|_{\infty}<\gamma$ with

$$
\left|L_{u^{*}}\right|_{\infty}
$$$$
=\sup _{v \in L_{F}^{2}\left([0, \infty), \mathbb{R}^{m_{1}}\right)}
$$

$$
\times \frac{\left\{\sum_{i=1}^{l} \lambda_{i} E\left[\int_{0}^{\infty}\left(x^{\mathrm{T}}(t) Q\left(r_{t}\right) x(t)+\left|u^{*}(t)\right|^{2}\right) \mathrm{d} t \mid r_{0}=i\right]\right\}^{1 / 2}}{\left\{\sum_{i=1}^{l} E\left[\int_{0}^{\infty}|v(t)|^{2} \mathrm{~d} t \mid r_{0}=i\right]\right\}^{1 / 2}}
$$

where $\lambda_{i}:=P\left(r_{0}=i\right)>0$ for all $i \in \varphi$.

Generally speaking, the $H_{\infty}$ control problem described by (9) and (10) is to find a control $u^{*}(t)$ such that $J\left(u^{*}\right)<0$ for arbitrary exogenous disturbances $v(t)$. As stated in [23], if we view $u(t)$ and $v(t)$ in the stochastic $H_{\infty}$ control problem as two control strategies of players $\mathrm{P}_{1}$ and $\mathrm{P}_{2}$ from the viewpoint of game theory, the $H_{\infty}$ control problem can be converted into solving a stochastic game problem, while $\left(u^{*}, v^{*}\right)$ is in fact the saddle point of this game, e.g.

$$
J\left(u^{*}, v\right) \leq J\left(u^{*}, v^{*}\right) \leq J\left(u, v^{*}\right)
$$

According to Theorem 1 discussed in Section 4, the following results can be obtained straightly:

Theorem 2. For system described by (9), the stochastic $H_{\infty}$ control admits a pair of solutions $\left(u^{*}, v^{*}\right)$ with $u^{*}(t)=K_{2}\left(r_{t}\right) x(t), v^{*}(t)=K_{1}\left(r_{t}\right) x(t)$, iff the following AREs

$$
\begin{aligned}
& P(i) A(i)+A^{\mathrm{T}}(i) P(i)+C^{\mathrm{T}}(i) P(i) C(i)+Q(i) \\
& +\sum_{j=1}^{l} \pi_{i j} P(j)-P(i) B(i) R^{-1}(i) B^{\mathrm{T}}(i) P(i)=0, i \in \varphi
\end{aligned}
$$

with

$$
B(i)=\left(B_{1}(i), B_{2}(i)\right), R(i)=\left(\begin{array}{cc}
-\gamma^{2} I & 0 \\
0 & I
\end{array}\right) .
$$

has a solution $P=(P(1), P(2), \cdots, P(l)) \in \mathrm{S}_{n}^{l}$, where

$$
\left\{\begin{array}{l}
K_{1}=\gamma^{-2} B_{1}^{\mathrm{T}}\left(r_{t}\right) P\left(r_{t}\right) \\
K_{2}=-B_{2}^{\mathrm{T}}\left(r_{t}\right) P\left(r_{t}\right)
\end{array} .\right.
$$

In this case, $u^{*}$ is an $H_{\infty}$ control for system (9), and $v^{*}$ is the corresponding worst case disturbance.

Illustrative example: Consider system (9) with the coefficients as follows:

$$
\varphi=\{1,2\}, \Pi=\left[\begin{array}{cc}
-1 & 1 \\
1 & -1
\end{array}\right], A(1)=\left[\begin{array}{cc}
-1 & 0 \\
1 & 1
\end{array}\right],
$$




$$
\begin{gathered}
A(2)=\left[\begin{array}{cc}
-1 & -1 \\
0 & 1
\end{array}\right], C(1)=\left[\begin{array}{cc}
-1 & 1 \\
0 & -2
\end{array}\right], \\
C(2)=\left[\begin{array}{cc}
-2 & 1 \\
1 & -1
\end{array}\right], B_{1}(1)=\left[\begin{array}{c}
1 \\
-1
\end{array}\right], B_{1}(2)=\left[\begin{array}{l}
1 \\
1
\end{array}\right], \\
B_{2}(1)=\left[\begin{array}{c}
-1 \\
1
\end{array}\right], B_{2}(2)=\left[\begin{array}{c}
-2 \\
1
\end{array}\right], Q(1)=\left[\begin{array}{ll}
1 & 2 \\
2 & 4
\end{array}\right], \\
Q(2)=\left[\begin{array}{ll}
4 & 2 \\
2 & 1
\end{array}\right] .
\end{gathered}
$$

Set $\gamma=100$, solving (11) via using the algorithm proposed in [21], we have

$P(1)=\left[\begin{array}{cc}9.5536 & 13.7815 \\ 13.7815 & 25.0974\end{array}\right], P(2)=\left[\begin{array}{cc}8.4178 & 16.5128 \\ 16.5128 & 44.1219\end{array}\right]$

Therefore, the $H_{\infty}$ control is given by

$u(t)=-4.2279 x_{1}(t)-11.3132 x_{2}(t)$ while $r_{t}=1$; $u(t)=0.3228 x_{1}(t)-11.0963 x_{2}(t)$ while $r_{t}=2$.

Remark 4. Although we restrict ourselves to single noise stochastic systems throughout the paper, our main theorem still hold for multiple multiplicative noise case. For example, if we replace (6) with

$$
\begin{aligned}
\mathrm{d} x(t)= & {\left[A\left(r_{t}\right) x(t)+B_{1}\left(r_{t}\right) u_{1}(t)+B_{2}\left(r_{t}\right) u_{2}(t)\right] \mathrm{d} t } \\
& +\sum_{k=1}^{\mathrm{d}} C_{k}\left(r_{t}\right) x(t) \mathrm{d} w_{k}(t), x(0)=x_{0}
\end{aligned}
$$

where $w_{k}, k=1,2, \cdots, \mathrm{d}$, being independent, one dimensional Wiener processes, then Theorem 1 still holds with AREs (7) replaced by

$$
\begin{aligned}
& P(i) A(i)+A^{\mathrm{T}}(i) P(i)+\sum_{k=1}^{\mathrm{d}} C_{k}^{\mathrm{T}}(i) P(i) C_{k}(i)+Q(i) \\
& +\sum_{j=1}^{l} \pi_{i j} P(j)-\left(P(i) B(i)+S^{\mathrm{T}}(i)\right) \\
& \times R^{-1}(i)\left(B^{\mathrm{T}}(i) P(i)+S(i)\right)=0, i \in \varphi
\end{aligned}
$$

with

$$
B(i)=\left(B_{1}(i), B_{2}(i)\right), S(i)=\left(\begin{array}{l}
S_{1}^{\mathrm{T}}(i) \\
S_{2}^{\mathrm{T}}(i)
\end{array}\right), R(i)=\left(\begin{array}{cc}
R_{1}(i) & 0 \\
0 & R_{2}(i)
\end{array}\right) .
$$

\section{Conclusion}

This paper has investigated the linear quadratic zero-sum stochastic differential games with state-dependent noise and Markovian jump parameters in infinite-time horizon, sufficient and necessary conditions for the existence of the optimal control strategies have been obtained, which are expressed in a system of coupled algebraic Riccati equations. The results obtained in this paper extend the existing results of [19]. Throughout this paper, we only have focused on the zero-sum LQ differential games for stochastic systems, while we believe that nonzero-sum LQ differential games still have essential applications, and further studies on such kind of case should be continued.

\section{Acknowledgements}

This work was supported by the National Natural Science Foundation of China under Grant No. 71171061, the Natural Science Foundation of Guangdong Province under Grant No. S2011010004970.

\section{REFERENCES}

[1] V. A. Ugrinovskii, "Robust $H_{\infty}$ Control in the Presence of Stochastic Uncertainty," International Journal of Control, Vol. 71, No. 2, 1998, pp. 219-237. doi:10.1080/002071798221849

[2] D. Hinrichsen and A. J. Pritchard, "Stochastic $H_{\infty}$, "SIAM Journal on Control and Optimization, Vol. 36, No. 5, 1998, pp. 1504-1538. doi: 10.1137/S0363012996301336

[3] B.-S. Chen and W. H. Zhang, "Stochastic $H_{2} / H_{\infty}$ Control with State-Dependent Noise," IEEE Transactions on Automatic Control, Vol. 49, No. 1, 2004, pp. 45-57. doi:10.1109/TAC.2003.821400

[4] Y. L. Huang, W. H. Zhang and G. Feng, "Infinite Horizon $\mathrm{H}_{2} / \mathrm{H}_{\infty}$ Control for Stochastic Systems with Markovian Jumps," Automatica, Vol. 44, No. 3, 2008, pp. 857-863. doi:10.1016/j.automatica.2007.07.001

[5] T. Basar and G. J. Olsder, "Dynamic Noncooperative Game Theory," 2nd Edition, SIAM, Philadelphia, 1999.

[6] E. J. Dockner, S. Jorgensen, N. Van Long and G. Sorger, "Differential Games in Economics and Management Science," Cambridge University Press, Cambridge, 2000. doi:10.1017/CBO9780511805127

[7] F. Avner, "Differential Games," Dover Publications, New York, 2006.

[8] S. Jorgensen and G. Zaccour, "Differential Games in Marketing," International Series in Quantitative Marketing, Kluwer Academic Publishers, London, 2004.

[9] T. L. Friesz, "Dynamic Optimization and Differential Games,” Springer, New York, 2009.

[10] E. K. Boukas, Q. Zhang and G. Yin, "Robust Production and Maintenance Planning in Stochastic Manufacturing Systems," IEEE Transactions on Automatic Control, Vol. 40, No. 6, 1995, pp. 1098-1102. doi:10.1109/9.388692

[11] T. Björk, "Finite Dimensional Optimal Filters for a Class of Itô-Processes with Jumping Parameters," Stochastics, Vol. 4, No. 2, 1980, pp. 167-183. doi:10.1080/17442508008833160

[12] D. D. Sworder, "Feedback Control of a Class of Linear Systems with Jump Parameters," IEEE Transactions on Automatic Control, Vol. 14, No. 1, 1969, pp. 9-14. doi:10.1109/TAC.1969.1099088

[13] V. Dragan and T. Morozan, "Stability and Robust Stabilization to Linear Stochastic Systems Described by Differential Equations with Markovian Jumping and Multi- 
plicative White Noise," Stochastic Analysis and Applications, Vol. 20, No. 1, 2002, pp. 33-92.

doi:10.1081/SAP-120002421

[14] V. Dragan and T. Morozan, "The Linear Quadratic Optimization Problems for a Class of Linear Stochastic Systems with Multiplicative White Noise and Markovian Jumping," IEEE Transactions on Automatic Control, Vol. 49, No. 5, 2004, pp. 665-675. doi:10.1109/TAC.2004.826718

[15] M. D. Fragoso and N. C. S. Rocha, "Stationary Filter for Continuous-Time Markovian Jump Linear Systems," SIAM Journal on Control and Optimization, Vol. 44, No. 3, 2006, pp. 801-815. doi:10.1137/S0363012903436259

[16] X. Li, X. Y. Zhou and M. A. Rami, "Indefinite Stochastic Linear Quadratic Control with Markovian Jumps in Infinite Time Horizon," Journal of Global Optimization, Vol. 27, No. 2-3, 2003, pp. 149-175. doi:10.1023/A:1024887007165

[17] X. R. Mao, G. G. Yin and C. G. Yuan, "Stabilization and Destabilization of Hybrid Systems of Stochastic Differential Equations", Automatica, Vol. 43, No. 2, 2007, pp. 264-273. doi:10.1016/j.automatica.2006.09.006

[18] A. S. Willsky, "A Survey of Design Methods for Failure Detection in Dynamic Systems," Automatica, Vol. 12, No.
6, 1976, pp. 601-611. doi:10.1016/0005-1098(76)90041-8

[19] M. McAsey and L. Mou, "Generalized Riccati Equations Arising in Stochastic Games," Linear Algebra and Its Applications, Vol. 416, No. 2-3, 2006, pp. 710-723. doi:10.1016/j.laa.2005.12.011

[20] M. A. Rami and X. Y. Zhou, "Linear Matrix Inequalities, Riccati Equations, and Indefinite Stochastic Linear Quadratic Controls," IEEE Transactions on Automatic Control, Vol. 45, No. 6, 2000, pp. 1131-1143. doi: $10.1109 / 9.863597$

[21] V. Dragan and I. Ivanon, "A Numerical Procedure to Compute the Stabilising Solution of Game Theoretic Riccati Equations of Stochastic Control,' International Journal of Control, Vol. 84, No. 4, 2011, pp. 783-800. doi: 10.1080/00207179.2011.578261

[22] Z. W. Lin, Y. Lin and W. H. Zhang, "A Unified Design for State and Output Feedback $H_{\infty}$ Control of Nonlinear Stochastic Markovian Jump Systems with State and Disturbance-Dependent Noise," Automatica, Vol. 45, No. 12, 2009 , pp. 2955-2962. doi:10.1016/j.automatica.2009.09.027

[23] W. H. Zhang and B.-S. Chen, "State Feedback $H_{\infty}$ Control for a Class of Nonlinear Stochastic Systems," SIAM Journal on Control and Optimization, Vol. 44, No. 6, 2006, pp. 1973-1991. doi: 10.1137/S0363012903423727 